\title{
True Brachial Artery Aneurysm Presenting as a Non-Pulsatile Mass
}

\author{
Anup Pradhananga, ${ }^{1} \mathrm{Xie}$ Chao' \\ 'Department of Radiodiagnosis (Ultrasound), Taihe Hospital, Hubei University of Medicine, Hubei, China.
}

\begin{abstract}
Brachial artery aneurysms are rare disease that can be encountered. It is divided into true and false. The frequency of true aneurysm of the brachial artery is so much unusual. So, we present a case of a 59 year old male who presented to us with complaint of mass in left upper limb since many years ago. Now, there was sudden onset of progressive pain with coldness, numbness, tingling sensation and blackish discolouration of skin from 8 hours. The left upper limb was pulseless and color Doppler ultrasound showed a non-pulsatile aneurysm at the mid level of left brachial artery associated with arterial occlusion in its distal branch by thrombus. The patient was successfully revascularised by interposing a saphenous vein graft. Thus, this case suggest that the prompt diagnosis of true brachial artery aneurysm by ultrasound or color Doppler ultrasound and the proper treatment by surgical repair can save limb from dangerous sequel
\end{abstract}

Keywords: brachial artery aneurysm; color doppler ultrasound; true aneurysm; ultrasound.

\section{INTRODUCTION}

Aneurysms are abnormal dilatations of the vessel wall that classified as true and false (Pseudo) types. True aneurysm is the localized dilation involving all three layers (intima, media and adventitia) of the arterial wall, but a false type or pseudo aneurysm is a collection of flowing blood that communicates with the arterial lumen and filled only by the adventitia or surrounding soft tissue. ${ }^{10}$ Upper extremity peripheral artery aneurysm like brachial artery aneurysms are rarely encountered in comparison to the lower extremity peripheral artery aneurysm like popliteal, femoral artery aneurysm.1-3 All brachial artery aneurysms are rare with an overall prevalence of $0.5 \% .{ }^{4}$ True aneurysm of the brachial artery is even more unusual for which we present such a case.

\section{CASE REPORT}

A 59 year old male referred to our radiodiagnosis department with complain of mass in left upper limb since many years ago. Now there was sudden onset of progressive pain with coldness, numbness, tingling sensation, blackish discolouration of skin in forearm and hand and decrease muscle force of his left upper limb from 8 hours. He had no significant history of other diseases. There was no history of trauma or medical procedures done in his left upper limb. He was non-smoker and non-alcoholic with no history of drug abuse. On physical examination, he had a swelling on his left arm with blackish discoloration of skin from middle of forearm to the hand. Palpation revealed a $20 \times 45 \mathrm{~mm}$ non-pulsatile mass with tenderness and the temperature was lower than surrounding skin. Pulse of the axillary artery was palpable but brachial, radial and ulnar arteries were pulseless from the site of swelling.

Correspondence: Dr. Anup Pradhananga, Deparment of Radiodiagnosis (Ultrasound), Taihe Hospital, Hubei University of Medicine, 30 South Renmin Road, Shiyan, Hubei, 442000, China. Email: anupladh@gmail.com, Phone: +8615926189402. 
Distal paresthesia was present. Cardiovascular system, chest and abdomen findings were normal.

On investigation, ultrasound examination of left upper limb showed a non-pulsatile, hypoechoic mass measuring $48 \times 25 \mathrm{~mm}$ with thin wall along the left brachial artery with intraluminal thrombosis from the area of swelling to the distal brachial artery course upto the radial and ulnar artery. Doppler's examination revealed no blood flow sound at the site of swelling. Color Doppler showed a hypoechoic non-pulsatile area measuring $48 \times 25 \mathrm{~mm}$ along left brachial artery and no flow from the area of swelling to the distal brachial artery course. The impression was of a true left brachial artery aneurysm.

For further investigation, we had suggested the patient and his relatives for the CT/MR angiography. But due to the high cost and patient could not afford it we had to depend on the USG and color Doppler USG finding. The USG and color Doppler USG findings was suggestive towards the true aneurysm that showed arterial wall and no visible blood flow within the mass which differentiate it from vascular tumor (Figure 1,2).

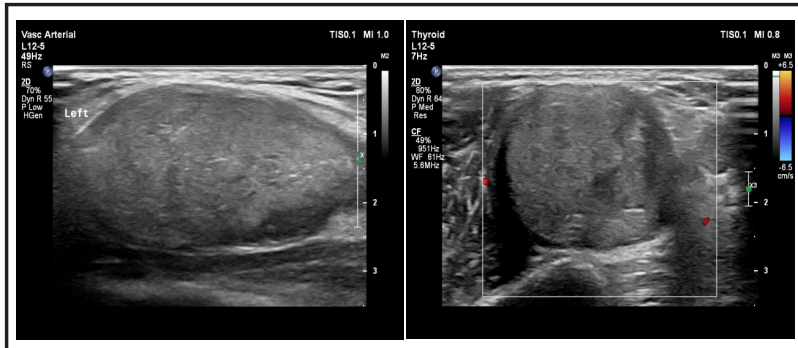

Figure 1. Imaging study. A) Ultrasound: longitudinal view showing brachial artery aneurysm with intraluminal thrombosis. B) Color Doppler ultrasound: transverse view showing brachial artery aneurysm with no flow due to intraluminal thrombosis.

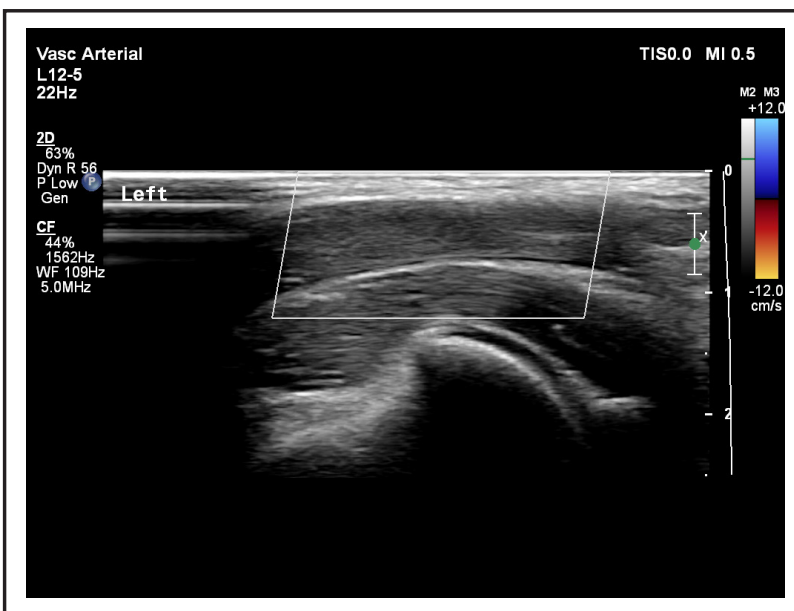

Figure 2. Imaging study. Color Doppler ultrasound: longitudinal view showing no flow in the radial artery due to intraluminal thrombosis.
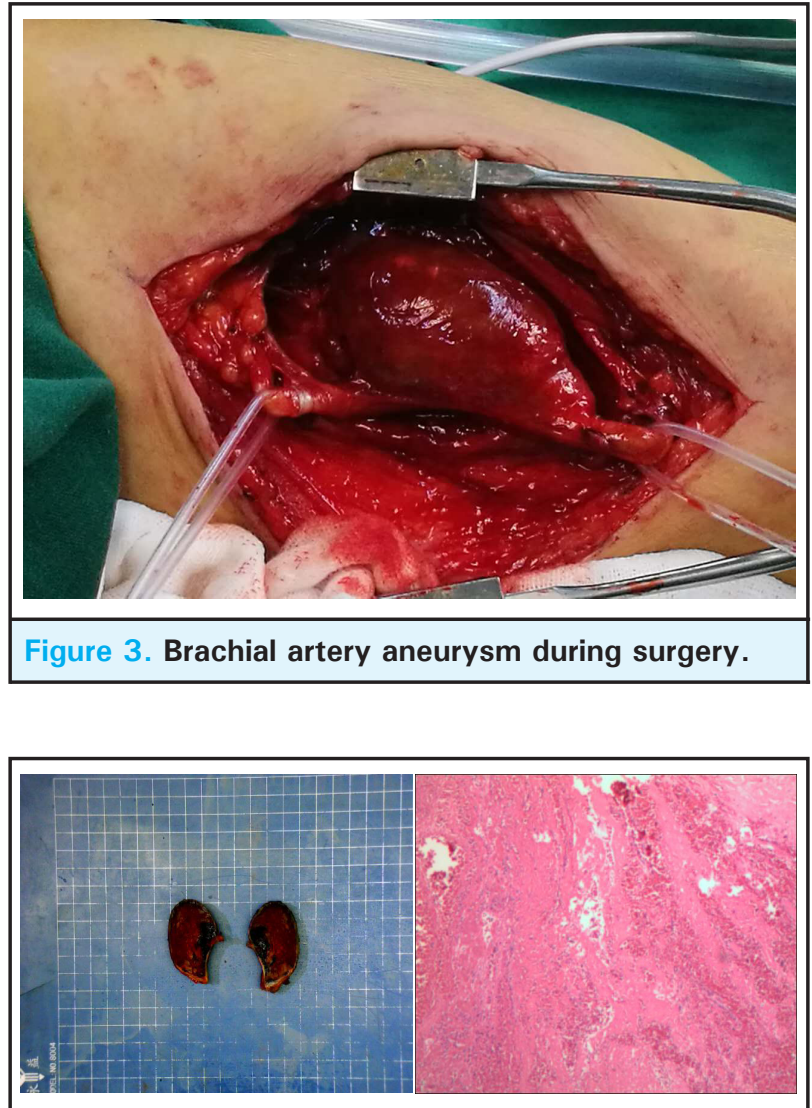

Figure 4. Histopathology study. A) Gross view of brachial artery aneurysm with thrombosis and uneven arterial wall. B) Microscopic view showing mainly thrombus component.

The patient was immediately referred to the surgery department as any delay in further intervention could lead to the ischaemic necrosis of limb resulting serious damage to the limb. The patient underwent surgery under general anesthesia. Intraoperatively, we found a saccular aneurysm originating from left brachial artery. Aneurysmal part of artery was resected and thrombus was evacuated by Forgarty catheter from brachial, radial and ulnar artery (Figure 3,4). After evacuation, 25,000 unit of urokinase was given. A reversed greater saphenous vein graft was interposed and anastomosed end to end proximally and distally to the normal remaining part of the brachial artery. The resected aneurysm displayed a true aneurismal sac filled with internal thrombus. After surgery the skin and temperature of arm and forearm were normal showing the blood flow. Elbow was positioned in flexion.

Histopathology showed a dilated arterial segment with thining of the arterial wall. There was evidence of mural thrombus formation with smooth muscle vitreous degeneration and calcification which had caused the arterial wall to balloon out. The diagnosis made by pathologist was True brachial artery 
aneurysm.

\section{DISCUSSION}

The dilatation of local segments of the arterial system is aneurysm. ${ }^{5}$ Brachial artery aneurysm can be of true or false nature. True aneurysms occur due to atherosclerotic process, repetitive trauma, congenital disease, inflammation or idiopathic. On the other hand, false aneurysms occur due to invasive procedures, infection and drug abuse. ${ }^{6}$ The usual manifestation of peripheral aneurysm is incidentally finding of pulsatile, painless and asymptomatic mass, which becomes symptomatic when complication arise. Thrombosis in the sac, vascular collapse which throws emboli into peripheral circulation, and also disruption caused the profuse bleeding are among these complications. ${ }^{2}$ The diagnosis should be confirmed by a duplex ultrasonography, which show the arterial blood flow into the aneurysm. Computed Tomography or magnetic resonance angiography can also be used for diagnosis. ${ }^{7}$ Inspite of all above mentioned, the gold standard tool is a selective upper extremity arteriography. However, the first choices are color Doppler ultrasonography and subtraction image angiography. ${ }^{8}$ Treatment options include observation, ultrasound guided compression, thrombin injection and operative repair. ${ }^{9}$
As in our case, a patient is 59 year old male, with nonpulsatile, tender aneurysm in brachial artery which is completely thombosed with no flow in color Doppler ultrasonography and the brachial artery successfully revascularised by interposing a saphenous vein graft.

Brachial artery aneurysms are rare. Moreover, true non-pulsatile aneurysms are even rarer. Brachial artery aneurysm could be limb threatening condition, so prompt diagnosis by ultrasound or color Doppler ultrasound and proper treatment can save limb from dangerous sequel. Surgical repair using autologous vein is the treatment of choice.

\section{ACKNOWLEDGEMENTS}

We thank Dr. Zhang Wen Jun and Dr. Bai Gang for supporting, Dr. Pan Jun Feng for surgery report, Dr. Long Binbin for pathological report and Dr. Moushami Singh, Dr. Sushil Kayastha and Dr. Rabin Gautam for motivating and helping me in writing this manuscript.

\section{Conflict of Interest: None.}

Consent: JNMA Case Report Consent Form was signed by the patient and the original is attached with the patient chart.

\section{REFERENCES}

1. Gray RJ, Stone WM, Fowl RJ, Cherry KJ, Bower TC. Management of true aneurysms distal to the axillary artery. J Vasc Surg. 1998;28(4):606-10. [Full Text | PubMed]

2. Tetik O, Ozcem B, Calli AO, Gurbuz A. True brachial artery aneurysm. Texas Heart Institute Journal. 2010;37(5):618-9. [PubMed]

3. Schunn CD, Sullivan TM. Brachial arteriomegaly and true aneurysmal degeneration: case report and literature review. Vasc Med. 2002;7(1):25-7. [PubMed]

4. Bahcivan M, Yuksel A. Idiopathic true brachial artery aneurysm in a nine month infant. Interact Cardiovasc Thorac Surg. 2009;8(1):162-3. [PubMed]

5. Ghazi MA, Khan AM, Akram Y, Cheema MA. Brachial artery aneurysm. JMAJ. 2006 Apr;49(4):173-5. [Full Text]
6. A Fakhree MB, Azhough R, Hafez Quran F. A case of true brachial artery aneurysm in an elderly male. J Cardiovasc Thorac Res. 2012;4(1):25-7. [PubMed | PMC | DOI]

7. Kouvelos GN, Papas NK, Arnaoutoglou EM, Papadopoulos GS, Matsagkas MI. Endovascular repair of profunda femoral artery false aneurysms using coveredstents. Vascular. 2011;19(1):51-4. [PubMed]

8. Yetkin U, Gurbuz A. Post-traumatic pseudoaneurysm of the brachial artery and its surgical treatment. Texas Heart Institute Journal. 2003;30(4):293-7. [PubMed]

9. Heydari F, Taheri M, Esmailian M. Brachial artery aneurysm as a limb threatening condition; a case report. Emergency. 2015;3(2):75-7. [PubMed]

10. Hall HA, Minc S, Babrowski T. Peripheral artery aneurysm. Surgical Clinics of North America. 2013;9(4):911-23. [PubMed] 